\title{
Genome-wide organization and expression profiling of the R2R3-MYB transcription factor family in sea buckthorn (Hippophae rhamnoides L.)
}

Hong Liu

Chinese Academy of Forestry Research Institute of Forestry https://orcid.org/0000-0002-3034-3763

Guoyun Zhang

Chinese Academy of Forestry Research Institute of Forestry

Zhongrui Lv

Chinese Academy of Forestry Research Institute of Forestry

\section{Songfeng Diao}

Chinese Academy of Forestry Research Institute of Forestry

\section{Caiyun He}

Chinese Academy of Forestry Research Institute of Forestry

Jianguo Zhang ( $\nabla$ zhangguoyun2012@163.com )

Chinese Academy of Forestry \& Nanjing Forestry University

\section{Research article}

Keywords: sea buckthorn, R2R3-MYB gene family, genome-wide analysis, gene expression pattern analysis, flavonoid biosynthesis

Posted Date: April 20th, 2020

DOl: https://doi.org/10.21203/rs.3.rs-18405/v1

License: (c) (i) This work is licensed under a Creative Commons Attribution 4.0 International License.

Read Full License 


\section{Abstract \\ Background}

MYB transcription factor family involved in multifarious stages of plant growth and development until death, which is one of the largest and most versatile gene families in plants, therefore it is an important regulatory factor in plants. Sea buckthorn (Hippophae rhamnoides L.) is rich in many secondary metabolites and has high nutritional and medicinal value. With the completion of sea buckthorn genome sequencing, it has laid the foundation for us to explore the gene structure, evolutionary relationship and function prediction of sea buckthorn MYB gene family from the whole genome perspective.

\section{Results}

In this study, 161 R2R3-MYB genes were identified from the sea buckthorn genome. We systematically analyzed their gene structure, collinearity, phylogenetic relationships and expression pattern. According to the gene structure, conserved motifs and phylogenetic relationship of $161 \mathrm{HrMYB}$ genes, all the HrMYB genes were divided into 28 subgroups. The phylogenetic tree of Hippophae rhamnoides L. and Arabidopsis thaliana R2R3-MYB genes showed that the sea buckthorn MYB gene family showed functional differentiation during evolution. Chromosomal localization results showed that the distribution of HrMYB genes were random. RNA-seq data from seven different tissues indicated that the HrMYB genes have significant spatiotemporal and tissue expression differences. QRT-PCR analysis showed that the selected genes which relate to flavonoid biosynthesis of sea buckthorn fruit expressed different degrees in different developmental stages of the fruit.

\section{Conclusion}

The sea buckthorn R2R3-MYB gene family was successfully identified from the whole genome sequence by a series of bioinformatics methods. The results firstly provide a more comprehensive insight for the phylogenetic relationships and expression patterns of the HrMYB genes. It will help us to further study their specific functions in the growth and development of sea buckthorn.

\section{Background}

In the process of growth, development, reproduction, death and coping with various stresses in plants, many genes are participated in regulating the expression of genes to control the biological processes. The difference in gene expression is often regulated by a variety of transcription factors as switches in cells[1]. At the N-terminus of transcription factor, there is a specific DNA binding domain that is translated into proteins and then bind to DNA, activating or inhibiting the expression of the gene[2]. Plants contain a variety of transcription factors, structurally, usually according to their DNA binding domain differences, can be divided into many different transcription factor gene families[3], such as MYB, WRKY, AP2, bHLH 
and NAC. MYB transcription factors are widely found in eukaryotes and are one of the most abundant and most versatile gene families in plants [4]. At the N-terminus, MYB gene has a highly conserved DNA binding domain characteristic. And each conserved domain sequence contains contiguous nonredundant incomplete repeats, each repeat having a length of approximately 52 amino acid residues. The MYB gene family members contain different numbers of repeat sequences, usually containing 1-4. The MYB gene, which contains two repeats, has the largest number of MYB members in plants, and constitutes the R2R3-MYB gene family[4, 5]. By contrast, activation domain of the MYB gene at the Cterminal isn't conserved, which may be closely related to the genetic diversity of the MYB gene family and its multiple regulatory roles[6, 7]. With the first MYB gene, C1, identified in Zea mays in 1987, more and more R2R3-MYB genes have been found in numerous plants[8]. So far, it has been confirmed that the R2R3-MYB genes are involved in various regulatory pathways in plant growth and development. Among them, the research on the Arabidopsis R2R3-MYB gene family is comprehensive[9]. In these studies, the R2R3-MYB gene family has diverse functions, including effects on cell fate progression, such as DhMYB1 affects the morphological development of conical cells of Dendrobium[10], DU01 in Arabidopsis affects the division and differentiation of male germ cells in Arabidopsis[11, 12]. It also effects on plant tissue formation and development, such as AtMYB118 and 115 co-regulating Arabidopsis embryogenesis[13] and RAX1 (AtMYB37) and RAX3 ( AtMYB84) regulates the formation of lateral meristems[14]. Moreover, MYB genes are potentially participated in plants responding to biotic and abiotic stresses, for example, TaMYB31 of wheat is involved in drought stress response[15], MdMYB88 and MdMYB124 in apples can enhance the cold tolerance[16]. In addition, R2R3-MYB gene can also regulate the synthesis and accumulation of plant secondary metabolites. And in more different plants, the R23R-MYB gene has a regulatory effect on different secondary metabolites including lignin, rutin, furanol and tanshinone. For instance, PpMYB10 in peach is a negative regulator involved in the accumulation of anthocyanins and proanthocyanidins[17] and AdMYB75 regulates the accumulation of chlorophyll and carotenoids in kiwifruit[18].

Sea buckthorn is a kind of tree with great economic benefits. It can be used as a pioneer plant for land vegetation restoration. Its fruit also has high nutritional value and is rich in various nutrients, including multivitamins $(A \backslash C \backslash K \backslash E)$, folic acid, various amino acids, phenols, ascorbic acid, carotenoids, and flavonoids, etc. Because of its high nutritional value and ecological value, sea buckthorn has attracted more and more people's attention. With the continuous updating of sequencing technology, there are more and more researches on plant genomes and transcriptomes. Studies on IncRNA in sea buckthorn revealed that it is involved in the induction of anthocyanin synthesis during fruit ripening[19], and the identification of circRNA associated with fruit development[20]. However, there are few researches on genome level of sea buckthorn. With the completion of the sequencing of the sea buckthorn genome, we can use the genomic information to identify the gene families, and provide a reference for further study. The R2R3-MYB gene family play a crucial regulatory role in all kinds of plants. Therefore, we chose to predict the conserved domains, gene structure, phylogeny relationship, chromosomal location and distribution, gene duplication and gene function of the R2R3-MYB gene family in sea buckthorn. RNA-seq data and phylogenetic relationship with Arabidopsis MYB genes were used to select the genes related to 
sea buckthorn fruit for real-time fluorescence quantitative PCR, so as to further determine the transcription level of these genes in fruit development, laying a solid foundation for further research on sea buckthorn fruit development.

\section{Results And Discussion}

\subsection{Identification 161 R2R3-MYB genes with conserved domains in the genome of sea buckthorn}

In order to accurately search genome-wide R2R3-MYB genes in sea buckthorn, we used Hmmsearch to search for gene sequences containing MYB conserved domain (PF00249). Finally, 230 MYB-related proteins were preliminarily identified in the sea buckthorn genome. According to the number of conserved domains, there were 161 R2R3-MYB proteins are selected. Among them, R2R3-MYB protein accounts for more than half of MYB protein, forming the largest number of MYB subfamilies [21]. According to the previous Arabidopsis MYB nomenclature[22], the 161 R2R3-MYB proteins were named HrMYB1 HrMYB161 (Additional file 1: Table S1). R2R3-MYB subfamily comprised $70 \%$ of sea buckthorn MYB genes and revealed that it was the largest MYB subgroup, which same with previous studies on cabbage[21].

Meanwhile, we used same methods to collected R2R3-MYB genes of 20 species from lower plants(algae, mosses) to higher plants(Angiospermae),such as Populus trichocarpa, Pyrus bretschneideri, Physcomitrella patens, Coccomyxa subellopsoidea and other plants in Additional file 2: Table S2. Among these species, higher plants have more R2R3-MYB genes than lower plants. All these results indicated that the R2R3-MYB family members experienced a huge expansion in the process of higher plants evolution.

\subsection{The classification, conserved domains, gene structure and motif composition of sea buckthorn R2R3-MYB gene family}

In order to investigate the characteristics of the homology domains, the R2 and R3 amino acid domains of 161 HrMYB proteins were analyzed their multiple sequence alignment (Fig. 1; Additional file 3: Table S3.). In general, the two conserved domains are approximately 97 amino acid residues and are highly conserved. According to previously reported, insertions or deletions in the two conserved domains were barely seen[21]. Consistent with other species, there are characteristic amino acids in the R2 and R3 amino acid repeats of sea buckthorn. The R2 repeat included three Trp (W) residues and the R3 repeat contained two $\operatorname{Trp}(\mathrm{W})$ residues. It was shown that those continuous $\operatorname{Trp}(\mathrm{W})$ residues play a vital role in ensuring the integrity of the helix-turn-helix structure of the R2R3-MYB gene [23]. Some hydrophobic amino acids, such as Phe (F), lle (I) and Leu (L), often replaced the first Trp residue of the R3 repeat in MYB,. Similarity, this substitution were also uncommonly observed at the third Trp residue of three HrMYBs (HrMYB2DHrMYB60 and HrMYB118) proteins R3 repeat. This change may affect HrMYB protein 
ability to recognize and directly combine DNA[24]. Not only contiguous Trp (W) residues in the MYB gene, but also many highly conserved amino acid residues in R2 and R3 repeats, such as Glu-8, Asp-9, Cys-44, Asn-50 in R2 repeat, and Glu-8, Gly-20 in R3 reprat. We also observed that Leu residue inserted into the R2 repeat of overwhelming majority of HrMYB proteins, which was different from MYB homologs in animal. This is an important step for the R2R3-MYB protein origin[25].

To gain the evolutionary relationship, we used MEGA7 to construct the ML phylogenetic tree for the amino acid sequence of $161 \mathrm{HrMYB}$ proteins (Figure. 2A). According to sequence similarity and topology, the HrMYBs were divided into 28 subgroups, of which ten genes appeared alone and didn't classified into any subgroup. The 28 subgroups were named A1-A28, and the number of genes in each subgroup was 2-12. Those with higher homology in the same subgroup.It is worth noting that there might be two or more HrMYB genes at the terminal nodes of each subgroup, which have high homology. This highly homologous protein might have similar functions[26]. In addition, there will also be low bootstrap support values in internal nodes, it's could be due to the large number of taxon and few available features. The phenomenon that internal nodes in the MYB gene family phylogenetic tree had low bootstrap support values had also been found in many animal and plant gene families. It was speculated that this might be due to the number of genes and the relatively small information

The gene structure of sea buckthorn R2R3-MYB genes were analyzed by TBTools. The results indicated that with the exception of 8 genes, the coding sequences of most HrMYB genes were separated by introns (Fig. 2B). The number of introns in the coding region of the MYB gene ranges from 0 to 12. The length of introns of some genes is much larger than coding region. Overall, approximately $70 \%$ of the DNA-binding domain of the sea buckthorn MYB gene contained three exons and two introns. According to the grouping information in the phylogenetic tree, the genes in the same group all showed similar gene structures. For example, all no introns genes in 20 group, and in the 28 group, the intron and exon distribution patterns are the same. Analysis of gene structure also provides important evidence for the classification of phylogenetic trees. The online software MEME was used to analyze the motifs of sea buckthorn R2R3MYB. Fifteen conserved motifs were finally identified from the HrMYB gene, which were designated as motif 1 to 15 (Fig. 2B; Additional file 4: Table S4; Additional file 5: Table S5). The results displayed that in the part outside the conserved domain, most MYB proteins sharing one or more motifs will be distributed in the same subgroup, showing that the structure of proteins in a specific subgroup was conservative (Fig. 2C). It is similar to the results of phylogenetic analysis, further indicating that the functions of those MYB genes clustered in the same subgroup might be similar[22]. As can be seen in Fig. 2B and 2C, it is indicated that the MYB gene in the same subgroup exhibited similar gene structures and conserved motif, which might have similar functions in the regulation of downstream target genes, and provided strong support for the possibility of MYB subfamily classification.

\subsection{Chromosome localization, replication events and collinearity analysis among sea buckthorn R2R3-MYB genes}


Chromosomal localization analysis of genes revealed that 161 R2R3-MYB candidate genes of sea buckthorn were distributed to 12 chromosomes, as shown in Fig. 3 and Additional file 6: Table S6. However, genes were not randomly distributed on all chromosomes and had a certain degree of bias. Among them, the largest number of MYB genes were distributed on chromosome 11, followed by chromosome 9 and chromosome 1, containing 27 and 22 MYB genes, respectively. In comparison, only two MYB genes are contained on chromosome 8. From the distribution pattern of genes in staining, the MYB genes of sea buckthorn were more concentrated on longer length chromosomes than shorter length chromosomes, such as chromosome $0,1,2,9,11$. In addition to the different numbers of distributions, the uniformity of distribution across chromosomes was also different. In some chromosomes, the region with high MYB gene density was found, which often form lots of clusters. For example, HrMYB137 and HrMYB138 located on chromosome 9 were contained on a 24 kb segment.

Gene duplication occurred during plant evolution, helping to derive genes with new functions, which is consistent with previous studies[27]. Multi-gene families formed were caused by the occurrence of genome-wide polyploidization or region-specific replication of chromosomes. Figure 4 showed total collinear relationship among all HrMYB genes were obtained using MCScanX. In the end, we found a total of 123 pairs of highly similar paralogous genes with highly similar protein sequences (Additional file 7: Table S7). Collinear gene pairs appeared on all chromosomes of sea buckthorn, simultaneously, the sea buckthorn genome also included the intrachromosomal duplication. If the distance between the two or more genes was within $100 \mathrm{~kb}$, which can be defined as a gene cluster[28]. Eight tandem duplications were identified in sea buckthorn (Additional file 6: Table S6). The number of segmental duplications in sea buckthorn was significantly higher than the number of tandem duplications, which had been found in many plants. This suggested that the occurrence of segment duplications might play a more critical role in the expansion of the sea buckthorn R2R3-MYB gene family[29].

\subsection{Comparative phylogenetic analysis of R2R3-MYB gene family between sea buckthorn and Arabidopsis}

To gain a deeper understanding for potential function of HrMYB gene family, 126 Arabidopsis and 161 sea buckthorn R2R3-MYB proteins were used to construct a combined ML phylogenetic tree (Fig. 5). Five HrMYB genes did not belong to any subgroups. This means that these MYB genes might have a special role in the evolution of the sea buckthorn genome[30]. The remaining MYB genes are divided into 38 subgroups with the number of MYB genes ranging from 2 to 18 . We also used the Arabidopsis R2R3-MYB gene classification model obtained in previous studies[7, 22] to classify the subgroup of the sea buckthorn R2R3-MYB genes. In the phylogenetic tree, some Arabidopsis genes classified in the same group in are still in the same group (e.g. C10, C13 and C21). The HrMYB genes was also mixed into the other Arabidopsis MYB gene groups (e.g. C1, C2 and C3). Counting the members of each subgroup, the number of HrMYBs was significantly more than AtMYBs. However, a small number of subgroups contain more AtMYBs than HrMYBs (e.g. C5, C7 and C8). It was worth noting that subgroups C16, C18, C32, C35 and C36 contain 3, 4, 2, 6 and 2 AtMYBs, respectively, and didn't contain HrMYBs. This might be due to a 
partial loss of function in evolution. In contrast, subgroups C15, C22 and C28 contained 6, 5 and 2 HrMYBs, respectively, and didn't contain AtMYBs. This might be part of the special role that sea buckthorn gained during evolution. These findings suggested that R2R3-MYB genes in sea buckthorn experienced not only duplication but also losting some gene functions after the differentiation of sea buckthorn and Arabidopsis[30]. In addition, the genomic drift may also cause the gene loss and lineagespecific amplification[31].

\subsection{Expression of HrMYB family genes in sea buckthorn}

In order to further understand the expression level of HrMYB gene, the spatial-temporal expression pattern analysis results of sea buckthorn R2R3-MYB gene family were obtained using transcriptome data of 7 different tissue and hierarchical clustering methods (Fig. 6, Additional file 8: Table S8). The results of the spatial-temporal expression pattern analysis showed that the expression profiles of HrMYB gene were significantly different in different tissue samples. Among them, $158 \mathrm{HrMYBs}$ were detected in at least one tissue, although some genes had low transcriptional abundance. Of these, 33 HrMYB genes were detected in 7 samples (FPKM $>1$ ) indicating that they might play a regulatory role in multiple developmental stages. At the same time, the transcriptional abundance of the three HrMYB genes (HrMYB72, HrMYB116 and HrMYB126) were not detected in either sample, which may indicate that the genes were pseudogenes of sea buckthorn in different tissues. Some genes tended to be expressed in specific tissues. For example, HrMYB6, HrMYB20 and HrMYB89 were only expressed in root nodule, stem and fruit, respectively. These genes might regulate special biological processes that occur only in specific tissues, and they were ideal candidates for subsequent functional analysis. In addition, the expression levels of some HrMYB genes in sea buckthorn fruits at different developmental stages are similar (e.g. HrMYB53, HrMYB76, HrMYB83 and HrMYB152), while others were different at different developmental stages, with high expression at one or two developmental stage and low or no expression at other stages. For example, HrMYB63, HrMYB73 and HrMYB89 were expressed in fruit1 and fruit2, but the expression level in fruit3 was very low. The genes HrMYB139, was higher expressed in fruit2 than in fruit1 and fruit3. Therefore, the results indicated that the expression of the sea buckthorn MYB gene family was spatiotemporal specific.

According to the phylogenetic analysis of sea buckthorn and Arabidopsis (Fig. 5), the function of sea buckthorn MYB genes could be inferred by comparing with the MYB gene that has been verified in model plants. AtMYB69 could reduce SCW thickening both in terfascicular fibers xylaryfibers[32]. HrMYB60 and AtMYB69 were closely related in the phylogenetic tree, and only had high expression in root, which indicated that this gene might also be involved in regulating the development of SCW in root. Interestingly, there were high expression of HrMYB42, HrMYB63, HrMYB120 and HrMYB160 in different tissues. The four genes were from subgroup $\mathrm{C} 1$ which is known that this subgroup was involved in the response of Arabidopsis to abiotic stress [33]. This indicated that subgroup C1 might play a role in responding to abiotic stress during the whole growth and development of sea buckthorn. In addition, the transcriptome data of HrMYB genes could also provide more information for further study of gene function. 


\subsection{Putative functions of the sea buckthorn R2R3-MYB gene}

Genes with similar functions usually belonged to homologous genes, and often clustered in the same clade or subclade in the phylogenetic tree[34]. However, paralogous genes usually exhibit different functional roles, which might be due to the fact that genes with similar functions can recognize similar target genes, and tihere might be also functional redundancy among them[35]. One of the most significant methods to predict the function of gene family was to identify the homologous HrMYB gene by phylogenetic analysis. By reason of Arabidopsis was a model species in plants, and all the gene of MYB had almostly studied. In particular, all 27 evolutionary branches of R2R3-AtMYBs had annotations[22, 36]. Therefore, in order to predict the function of the HrMYBs, a co-constructed ML phylogenetic tree by R2R3-MYB proteins of Arabidopsis and Hippophae rhamnoides were utilized in our study (Fig. 5). By comparing with the known functional model plant Arabidopsis MYB gene, the potential function of each HrMYB gene can be further predicted. Genes from the same subgroup sharing the same motif might play similar roles during plant growth and development[22].

The first category of MYB genes mainly play an important role in controlling of tissue traits, morphogenesis and organogenesis during plant growth and development, such as plant cells, anthers, stomatal cell development and embryogenesis, including six clades (respectively S9, S14, S15, S18, S19, S21, S25). Different types of Arabidopsis epidermal cells were affected by the MYB gene. However, the MIXTA-like transcription factor AtMYB106 of the S9 subgroup had a negative regulatory effect on the trichome branch of Arabidopsis roots[37]. HrMYB34 which belong to the same group as S9(C35) were also presumed to have similar functions. In addition, AtMYB21 and AtMYB24 (S19) and AtMYB33 and AtMYB65 (S18) jointly control the development and function of Arabidopsis anthers and pollen[38-40]. Based on the functions of these two clades of At-MYBs, it is reasonable to assume that HrMYB77, HrMYB123, HrMYB53, HrMYB3Which were in the same branch might have similar functions.

The second category is mainly responsible for regulating the stress response of plants to biotic and abiotic stresses, and helping plants to adapt to environmental unfavorable conditions, mainly distributed in seven subgroups (S1, S2, S11, S15, S18, S20, S22). The three proteins in subgroup 1 (AtMYB30, AtMYB60 and AtMYB96) have a certain stress response to both biotic and abiotic stresses and protect plants to grow and develop normally [41-44]. AtMYB15 in S2 was involved in allowing plants to achieve low temperature tolerance[45]. Two MYB genes (AtMYB102 and AtMYB41) located in S11 enhanced Arabidopsis resistance to insects[46, 47]. Many HrMYB proteins belonging to these six groups may also help plants to resist biotic and abiotic stresses and better adapt to the environment.

The third type of MYB gene mainly regulated the synthesis of plant secondary metabolites, including eight subgroups (S2, S3, S4, S5, S6, S7, S12, S31). Sea buckthorn was an important economic tree species, which fruits and leaves were rich in many secondary metabolites, among which flavonoids compounds had the most nutritional value. Flavonoids is a kind of crucial secondary metabolites, which is important for people's health and has potential medicinal value. The members of the three subgroups S2, S4,S6, and S7 played a regulatory role in the synthesis of different flavonoid compounds. AtMYB123 
in S2 regulates the biosynthesis of proanthocyanidins (PAs) in Arabidopsis seed coats[48], and four genes in $\mathrm{S} 6$ are involved in the regulation of anthocyanin synthesis in vegetative tissues[49]. The synthesis of flavonols can be regulated in all tissues by AtMYBs in the 57[50]. The MYB proteins of the sea buckthorn clustered in the phylogenetic tree with the three subgroups have similar conserved motifs to the Arabidopsis MYB protein, suggesting that these HrMYB genes had the possibility involved in regulating flavonoid synthesis. AtMYB4 in subgroup 4, a transcriptional repressor, is regulated by aUV to regulate the biosynthesis of intracellular gluconate[51]. HrMYB22 and AtMYB4 were on the same terminal branch and had similar genetic relationships, so it is speculated that they may have similar functions. There were many HrMYB genes in these clades to regulate the synthesis and accumulation of secondary metabolites in itself.

In conclusion, according to the R2R3-MYB gene in Arabidopsis, it is speculated that R2R3-MYB of sea buckthorn also has a wide range of functions. They jointly affected the expression of tissue traits, morphogenesis and organogenesis, the production and accumulation of secondary metabolites in the growth and development of sea buckthorn, and helped plants cope with various biotic and abiotic stresses. So that plants could better adapt to the environment and maintain normal growth and development. The specific functions of the different sea buckthorn R2R3-MYB genes need to be further verified later.

\subsection{Verification of HrMYB gene expression by QRT-PCR}

The fruit of sea buckthorn is a kind of berry, rich in secondary metabolites, which is the most nutritious and economic value part. Therefore, to further verify the expression pattern of R2R3-MYB genes related to flavonoid biosynthesis during different fruit development, 14 HrMYB genes that might be closely related to flavonoid biosynthesis were selected. Then, their expression levels in five different developmental stages of fruits were determined by real-time quantitative PCR. Further explore the changes of transcript abundance of 14 genes in different developmental stages of fruits, and provide reference for subsequent functional verification. The results were obtained using QRT-PCR showed that the expression pattern of $14 \mathrm{HrMYB}$ genes is closely associated with the change of flavonoids during the fruit development of sea buckthorn (Fig. 7; Additional file 9: Table S9). The results showed that some genes peaked only early in fruit development, such as HrMYB8, HrMYB9, HrMYB23, HrMYB27, HrMYB114 peaked at first stage and HrMYB110 which peaked at second stage. The expression levels of gene HrMYB157 peaked at first stage and gene HrMYB104 peaked at second stage, subsequently, their expression levels gradually decreased with fruit development. This trend was in line with the change of flavonoids content during sea buckthorn fruit development[52]. In addition, gene HrMYB11, HrMYB14, HrMYB19, HrMYB107 and HrMYB58 had higher expression levels at the first stage and lower at the second or third stage. Subsequently, the expression levels significantly up-regulated, expect for the HrMYB58, which the expression level had declined at the fifth stage. Consequently, we guessed that they might be negative regulators of flavonoids synthesis during sea buckthorn fruit development.[53]. In particular, the expression level of gene HrMYB80 showed a tendency of increasing at first stage and reaching a peak at the third stage and then 
decreasing with fruit development. Thus, we speculated the gene HrMYB80 may play a special role at the third stage of sea buckthorn fruit development.

Because the MYB transcription factors between herb and xylophyta have complex functions, the functional predictions performed in this paper may not be completely correct, and subsequent functional verification is needed to further determine their specific functions during plant growth and development.

\section{Material And Methods}

\subsection{Identification the R2R3-MYB family genes in sea buckthorn and physicochemical property of proteins}

The genome data of sea buckthorn were obtained from our research group and have not been published yet, which strongly promoted the whole genome analysis of the sea buckthorn gene family. From the Pfam database (http://pfam.xfam.org) [54], download the HMM (Hidden Markov Model) of the MYB DNA-binding domain (PF00249) and hmmsearch was used to search the sequences of sea buckthorn containing MYB domain in the genome of sea buckthorn. Protein sequences only with e-value $<1 e-10$ were selected to form the putative MYB gene family[21]. Then we used TBtools to extract the putative MYB sequence from the genome. According to the comparison results of Pfam online operation, R2R3MYB sequences containing two conserved domains were screened from the assumed MYB sequences, and failure to meet the terms of R2R3-MYB sequences were got rid of the putative MYB sequences according to the alignment results.

\subsection{Structure analysis of HrMYB genes}

To analyze the conserved DNA-binding domains of the sea buckthorn R2R3-MYB proteins, we first need to use the ClustalW program in the Linux system to conduct multiple sequence comparison of all HrR2R3MYB protein sequences, thus The amino acid sequence differences between R2 and R3 MYB were deduced. Then using WEBLOGO online program(http://weblog.berkeley.edu/logo.cgi) to display the distribution of amino acid residues in the R2 and R3 repeats of the MYB gene T [55]. Using the online MEME program (version 4.8.1) (http://meme.nbcr.net/meme/intro.html), we can identification and statistics the conserved motifs of 161sea buckthorn R2R3-MYB proteins[56]. The settings of the personalized parameters were as follows: the width of each conserved motif sequence was 10-200 amino acids and the number of conserved motifs was set to 20. GSDS (Gene Structure Display Server) (http://gsds.cbi.pku.edu.cn/)[57] was employed to clarify exon and intron structure of the HrMYB genes.

\subsection{Analyses of chromosomal locations, duplications and synteny analysis for all HrMYB genes}

To obtain the chromosomal mapping of all HrMYB genes, We extracted their corresponding physical location and chromosome length from the genomic database of sea buckthorn. Then we employed Maplnspect to draw the positions of each HrMYB gene on chromosomes and using 
mapGene2Chromosome to adjust and beautify the result. Utilizing the Multiple Collinearity Scan toolkit (MCscanX) and following the instructions with default settings to analyze the duplication pattern and syntenic relationship for HrMYB gene [58].

\subsection{Phylogenetic analysis $₫$ classification and function prediction of the HrMYB genes family}

To further analyze the evolutionary relationship of 161 sea buckthorn R2R3-MYBs, classify MYBs and predict their functions, the clustaW the in Linux system was employed to aligned the full length amino acid sequences of $161 \mathrm{HrMYBs}$ and 126 AtMYBs[59]. Then a ML phylogenetic tree with full-length R2R3MYB genes from sea buckthorn and Arabidopsis was constructed using MEGA 7.0. At the same time set the parameters to poson correction and bootstrap 1000 repeated analyses. In the construction of $\mathrm{ML}$ phylogenetic tree, the same method was used to generate phylogenetic tree separately from the fulllength sequence of sea buckthorn R2R3-MYB TFs. In addition, the phylogenetic tree constructed by sea buckthorn and Arabidopsis can also predict the biological function of HrMYB protein based on homology, and the specific functions of homologous Arabidopsis proteins have been verified in the published literatures[60-62].

\subsection{RNA-seq data analysis of seabuckthorn R2R3-MYB gene family}

To investigate the R2R3-MYB genes expression patterns of in seven different tissues of sea buckthorn, we used transcriptome data for the fruits,leaves $\square$ stems $\square$ root nodule and roots, which were derived from previously published RNA-seq raw data[19]. Cuffdiff was used to calculate the expression level of each MYB gene, and the expression level was estimated by the thousand-base fragment (FPKM) value per million mapped reads. Using the Cluster 3.0 to hierarchical clustered the expression data and used $\mathrm{R}$ language to generated heat map the heat map.

\subsection{RNA isolation and quantitative real-time RT-PCR (QRT- PCR)}

The 'Hippophae rhamnoides subsp. mongolica' as the research object in this experiment were collected from the desert forest experimental centre in Inner Mongolia, China. We chose three biological replicates of sea buckthorn samples and harvested the healthy fresh fruit at five different developmental stages (S1-S5) from May to July. The fruit samples were collected at 21, 49, 63, 77 and 91 days post-anthesis. All fruit samples were quick-frozen with liquid nitrogen after collection and stored at $-80{ }^{\circ} \mathrm{C}$. Total RNA was extracted from sea buckthorn fruits at 5 different developmental stages by using the procedure of the plant RNA extraction kit (Qiangen, RNA prep Pure Plant Kit (Polysaccharides \& Polyphenolics-rich). Verify the quality of the extracted total RNA by using agarose gel electrophoresis, and then the highly quality RNA was selected for the next experiment. At the same time, their purity and concentration were measured by Nano-drop 2000. Total RNA was immediately frozen with liquid nitrogen and stored at $-80^{\circ}$ $\mathrm{C}$ before used. DNase I was used to remove the remaining DNA in the total RNA. First strand cDNA was 
synthesized using a reverse transcription system of Takara (PrimeScript ${ }^{\text {TM }}$ RT reagent Kit with gDNA Eraser (Perfect Real Time)). According to the reaction procedure in the instruction manual, each $20 \mu \mathrm{l}$ reflecting system contained $1,000 \mathrm{ng}$ of total RNADoligo-dT primers and reverse transcriptase. The reaction program was set to 15 minutes at $37^{\circ} \mathrm{C}$ and 5 seconds at $85^{\circ} \mathrm{C}$.

Based on the design principle of real-time fluorescence quantitative PCR primer, specific primers for 14 selected HrMYBs were used Primer Premier 5.0 software to design, and the specificity of the primers was according to the results of preliminary experiments. All Primer sequences in this study are detailed in the Additional file 10: Table S10. Three biological replication of fruit samples were used for qPCR assays. QRT-PCR was performed using TB Green ${ }^{\circledR}$ Premix Ex Taq ${ }^{\text {TM }}$ II (Tli RNaseH Plus) of Takara on Roche Lightcyler ${ }^{\circledR} 480$ instrument. The endogenous control gene 18S rRNA was selected as the reference gene. The hot-start cycle curve of the reactions is as follows: $95^{\circ} \mathrm{C}$ for $30 \mathrm{sec}$, followed by 40 cycles at $95^{\circ} \mathrm{C}$ for $5 \mathrm{sec}$ and $60{ }^{\circ} \mathrm{C}$ for $30 \mathrm{sec}$. The reaction volume was $20 \mu \mathrm{l}$, and using the using the $2^{-\triangle \triangle \mathrm{CT}}$ method to calculation and visualization of real-time PCR data generated[63].

\subsection{Statistical analysis of experimental data}

SPSS software was used for statistical analysis of the experimental data. All the calculated average and standard error were derived from the data of three repeated experiments. SPSS software was used for one-way analysis of variance and evaluated the statistical significance of mean difference.

\section{Conclusion}

MYB gene family is one of biggest TFs family in higher plant and play a critical role in growth and development of plants. In this study, 161 R2R3-MYB genes were successfully identified from sea buckthorn genome and divided into 28 subfamilies based on their phylogenetic relationships, genetic structure and conservative motif composition. All these R2R3-MYB genes randomly distributed on 12 chromosomes of sea buckthorn. The function of HrMYBs was predicted based on the phylogenetic relationship with Arabidopsis and compared with the identified functional AtMYBs. In particular, the function of 14 HrMYBs related to flavonoid biosynthesis pathway was verified by qRT-PCR. The results demonstrated that the abundance level of their transcript changed significantly as the fruits matured. The results of this work provided a reference for the further study of sea buckthorn R2R3-MYB transcription factors, and also laid the foundation for the subsequent study of the biological role of HrMYB genes.

\section{Declarations}

Ethics approval and consent to participate

Not applicable

Consent for publication

Not applicable. 
Availability of data and materials

All data generated or analyzed during this study are included in this published article and its supplementary information files.

Competing interests

The authors declare that they have no competing interests.

Funding

This work was supported by the Fundamental Research Funds of CAF (CAFYBB2020SY006). The funding agency provided financial support for this research, but had no role in the research design, data collection, analysis, interpretation, and writing of the manuscript.

Author Contributions

H.L., G.Z. and

J.Z. initiated and designed the research. H.L., G.Z., and Z.L. performed the experiments. G.Z. and H.L. analyzed the data. H.L. and Z.L. prepared materials. H.L. and G.Z. wrote the manuscript. C.Z. and S.D. provided the experimental materials.

All of the authors read and approved the final manuscript. All authors have read and agreed to the published version of the manuscript.

Acknowledgments

Not applicable.

Authors' Information

Hong Liu and Guoyun Zhang contributed equally to this work.

\section{References}

1. Scott MP: Development: The natural history of genes. Cell 2000, 100(1):27-40.

2. Riechmann JL, Heard J, Martin G, Reuber L, Jiang C-Z, Keddie J, Adam L, Pineda O, J.Ratcliffe O, Samaha RR et al: Arabidopsis Transcription Factors: Genome-Wide Comparative Analysis Among Eukaryotes. Science 2000, 290(5499):2105-2110.

3. Luscombe NM, Austin SE, Berman HM, Thornton JM: An overview of the structures of protein-DNA complexes. Genome Biology 2000, 1(1):1-37.

4. Martin C, Paz-Aers J: MYB transcription factors in plants. Trends in Genetics 1997, 13(2):67-73.

5. Nagano Y: Several Features of the GT-Factor Trihelix Domain Resemble Those of the Myb DNABinding Domain. Plant Physiology 2000, 124(2):491-493. 
6. Du H, Wang Y-B, Xie Y, Liang Z, Jiang S-J, Zhang S-S, Huang Y-B, Tang Y-X: Genome-Wide Identification and Evolutionary and Expression Analyses of MYB-Related Genes in Land Plants. DNA Research 2013, 20(5):437-448.

7. Niu Y, Jiang X, Xu X: Reaserch Advances on Transcription Factor MYB Gene Family in Plant. Molecular Plant Breeding 2016, 14(8):2050-2059.

8. Paz-Ares J, Ghosal D, Wienand U, A.Petersont P, Saedler H: The regulatory $\mathbf{c} 1$ locus of Zea mays encodes a protein with homology to myb proto-oncogene products and with structural similarities to transcriptional activators. Embo Journal 1987, 6(12):3553-3558.

9. Feng P-p, Chen P, Hong W-j, Zhao X-y, Liu X-m: Research Progress of MYB Transcription Factor Family in Arabidopsis thaliana. Life Science Research 2016, 20(6):555-560.

10. Lau S-E, Schwarzacher T, Othman RY, Harikrishna JA: dsRNA silencing of an R2R3-MYB transcription factor affects flower cell shape in a Dendrobium hybrid. Bmc Plant Biology 2015, 15(194):1-14.

11. Borg M, Brownfield L, Khatab H, Sidorova A, Lingaya M, Twell D: The R2R3 MYB transcription factor DU01 activates a male germline-specific regulon essential for sperm cell differentiation in Arabidopsis. The Plant Cell 2011, 23(2):534-549.

12. Brownfield L, Hafidh S, Borg M, Sidorova A, Mori T, Twell D: A Plant Germline-Specific Integrator of Sperm Specification and Cell Cycle Progression. Plos Genetics 2009, 5(3):e1000430.

13. Wang X, Niu Q-W, Teng C, Li C, Mu J, Chua N-H, Zuo J: Overexpression of PGA37/MYB118 and MYB115 promotes vegetative-to-embryonic transition in Arabidopsis. Cell Research 2009, 19(2):224235.

14. Keller T, Abbott J, Moritz T, Doerner P: Arabidopsis regulator of Axillary Meristems1 Controls a Leaf Axil Stem Cell Niche and Modulates Vegetative Development. Plant Cell 2006, 18(3):598-611.

15. Zhao Y, Cheng X, Liu X, Wu H, Bi H, Xu H: The Wheat MYB Transcription Factor TaMYB31 Is Involved in Drought Stress Responses in Arabidopsis. Frontiers in plant science 2018, 9:1-12.

16. Geng D, Chen P, Shen X, Zhang Y, Li X, Jiang L, Xie Y, Niu C, Zhang J, Huang X et al: MdMYB88 and MdMYB124 Enhance Drought Tolerance by Modulating Root Vessels and Cell Walls in Apple. Plant Physiology 2018, 178(3):1296-1309.

17. Tuan PA, Bai S, Yaegaki H, Tamura T, Hihara S, Moriguchi T, Oda K: The crucial role of PpMYB10.1 in anthocyanin accumulation in peach and relationships between its allelic type and skin color phenotype. Bmc Plant Biology 2015, 15(1):1-14.

18. Li W, Ding Z, Ruan M, Yu X, Peng M, Liu Y: Kiwifruit R2R3-MYB transcription factors and contribution of the novel AcMYB75 to red kiwifruit anthocyanin biosynthesis. Scientific Reports 2017, 7(1):1-14.

19. Zhang G, Chen D, Zhang T, Duan A, Zhang J, He C: Transcriptomic and functional analyses unveil the role of long non-coding RNAs in anthocyanin biosynthesis during sea buckthorn fruit ripening. Dna Research An International Journal for Rapid Publication of Reports on Genes \& Genomes 2018, 25(5):465-476.

20. Zhang G, Diao S, Zhang T, DaoguoChen, He C, Zhang J: Identification and characterization of circular RNAs during the sea buckthorn fruit development. RNA Biology 2019, 16(3):354-361. 
21. Wang Z, Tang J, Hu R, Wu P, Hou X-L, Song X-M, Xiong A-S: Genome-wide analysis of the R2R3-MYB transcription factor genes in Chinese cabbage (Brassica rapa ssp. pekinensis) reveals their stress and hormone responsive patterns. Bmc Genomics 2015, 16(1):1-21.

22. Dubos C, Stracke R, Grotewold E, Weisshaar B, Martin C, Lepiniec Lc: MYB transcription factors in Arabidopsis. Trends in Plant Science 2010, 15(10):573-581.

23. Saikumar P, Murali R, Reddy EP: Role of tryptophan repeats and flanking amino acids in Myb-DNA interactions. Proceedings of the National Academy of Sciences of the United States of America 1990, 87(21):8452-8456.

24. Dias AP, Braun EL, McMullen MD, Grotewold E: Recently Duplicated Maize R2R3 Myb Genes Provide Evidence for Distinct Mechanisms of Evolutionary Divergence after Duplication. Plant Physiology 2003, 131(2):610-620.

25. Rabinowicz PD, Braun EL, Wolfe AD, Bowen B, Grotewold E: Maize R2R3 Myb Genes: Sequence Analysis Reveals Amplification in the Higher Plants. Genetics 1999, 153(1):427-444.

26. Pan1 F, Wang1 Y, Liu H, Wu M, Chu W, Chen D, Xiang Y: Genome-wide identification and expression analysis of SBP-like transcription factor genes in Moso Bamboo (Phyllostachys edulis). BmC Genomics 2017, 18(1):1-17.

27. Cannon SB, Mitra3 A, Baumgarten A, Young ND, May G: The roles of segmental and tandem gene duplication in the evolution of large gene families inArabidopsis thaliana. BMC Plant Biology 2004, 4(1):1-21.

28. Huang S, Gao Y, Liu J, Peng X, Niu X, Fei Z, Cao S, Liu Y: Genome-wide analysis of WRKY transcription factors in Solanum lycopersicum. Molecular Genetics \& Genomics Mgg 2012, 287(6):495-513.

29. Liu C, Xie T, Chen C, Luan A, Long J, Li C, Ding Y, He Y: Genome-wide organization and expression profiling of the R2R3-MYB transcription factor family in pineapple (Ananas comosus). BmC Genomics 2017, 18(1):1-16.

30. Zhao P, Li Q, Li J, Wang L, Ren Z: Genome-wide identification and characterization of R2R3MYB family in Solanum lycopersicum. Molecular Genetics \& Genomics 2014, 289(6):1183-1207.

31. Nozawa M, Kawahara Y, Nei M: Genomic drift and copy number variation of sensory receptor genes in humans. Proceedings of the National Academy of Sciences of the United States of America 2007, 104(51):20421-20426.

32. Zhong R, Lee C, Zhou J, McCarthy RL, Ye Z-H: A Battery of Transcription Factors Involved in the Regulation of Secondary Cell Wall Biosynthesis in Arabidopsis. Plant Cell 2008, 20(10):2763-2782.

33. Jung C, Seo JS, Han SW, Koo YJ, Kim CH, Song SI, Nahm BH, Choi YD, Cheong J-J: Overexpression of AtMYB44 Enhances Stomatal Closure to Confer Abiotic Stress Tolerance in Transgenic Arabidopsis. Plant Physiology 2007, 146(2):623-635.

34. Fitch WM: Distinguishing homologous from analogous proteins. Systematic zoology 1970, 19(2):99113. 
35. Minoru K, Susumu G: KEGG: Kyoto Encyclopedia of Genes and Genomes. Nucleic Acids Research 2000, 28(1):27-30.

36. Li X, Xue C, Li J, Qiao X, Li L, Yu La, Huang Y, Wu J: Genome-Wide Identification, Evolution and Functional Divergence of MYB Transcription Factors in Chinese White Pear (Pyrus bretschneideri). Plant \& cell physiology 2016, 57(4):824-847.

37. Jakoby MJ, Falkenhan D, Mader MT, Brininstool G, Wischnitzki E, Platz N, Hudson A, Hulskamp M, Larkin J, Schnittger A: Transcriptional Profiling of Mature Arabidopsis Trichomes Reveals That NOECK Encodes the MIXTA-Like Transcriptional Regulator MYB106. Plant Physiology 2008, 148(3):1583-1602.

38. Millar AA, Gubler F: The Arabidopsis GAMYB-Like Genes, MYB33 and MYB65, Are MicroRNARegulated Genes That Redundantly Facilitate Anther Development. The Plant Cell 2005, 17(3):705721.

39. Mandaokar A, Browse J: MyB108 Acts Together with MyB24 to Regulate Jasmonate-Mediated Stamen Maturation in Arabidopsis. Plant Physiology 2008, 149(2):851-862.

40. Cheng H, Song S, Xiao L, Soo HM, Cheng Z, Xie D, Peng J: Gibberellin Acts through Jasmonate to Control the Expression of MYB21, MYB24, and MYB57 to Promote Stamen Filament Growth in Arabidopsis. Plos Genetics 2009, 5(3):e1000440.

41. Raffaele S, Vailleau F, Le'ger A, Joube`s Jrm, Miersch O, Huard C, Ble'e E, Mongrand Sb, Domergue Fdr, Roby D: A MYB Transcription Factor Regulates Very-Long-Chain Fatty Acid Biosynthesis for Activation of the Hypersensitive Cell Death Response in Arabidopsis. Plant Cel/ 2008, 20(3):752-767.

42. Cominelli E, Galbiati M, Vavasseur A, Conti L, Sala T, Vuylsteke M, Leonhardt N, Dellaporta SL, Tonelli C: A Guard-Cell-Specific MYB Transcription Factor Regulates Stomatal Movements and Plant Drought Tolerance. Current Biology 2005, 15(13):1196-1200.

43. Seo PJ, Xiang F, Qiao M, Park J-Y, Lee YN, Kim S-G, Lee Y-H, Park WJ, Park C-M: The MYB96 Transcription Factor Mediates Abscisic Acid Signaling during Drought Stress Response in Arabidopsis. Plant Physiology 2009, 151(1):275-289.

44. Seo PJ, Park C-M: MYB96-mediated abscisic acid signals induce pathogen resistance response by promoting salicylic acid biosynthesis in Arabidopsis. New Phytologist 2010, 186(2):471-483.

45. Agarwal M, Hao Y, Kapoor A, Dong C-H, Fujii H, Zheng X, Zhu J-K: A R2R3 Type MYB Transcription Factor Is Involved in the Cold Regulation of CBF Genes and in Acquired Freezing Tolerance*. Journal of Biological Chemistry 2006, 281(49):37636-37645.

46. Vos MD, Denekamp M, Dicke M, Vuylsteke M, Loon LV, Smeekens SC, Pieterse C: The Arabidopsis thaliana Transcription Factor AtMYB102 Functions in Defense Against The Insect Herbivore Pieris rapae. Plant Signaling \& Behavior 2006, 1(6):305-311.

47. Lippold F, Sanchez DH, Musialak M, Schlereth A, Scheible W-R, Hincha DK, Udvardi MK: AtMyb41 Regulates Transcriptional and Metabolic Responses to Osmotic Stress in Arabidopsis. Plant Physiology 2009, 149(4):1761-1772. 
48. Lepiniec LI, Debeaujon I, Routaboul J-M, Baudry A, Pourcel L, Nesi N, Caboche M: Genetics and Biochemistry of Seed Flavonoids. Annual Review of Plant Biology 2006, 57(1):405-430.

49. Gonzalez A, Zhao M, Leavitt JM, Lloyd AM: Regulation of the anthocyanin biosynthetic pathway by the TTG1/bHLH/Myb transcriptional complex in Arabidopsis seedlings. Plant Journa/2008, 53(5):814-827.

50. Stracke R, Ishihara H, Huep G, Barsch A, Mehrtens F, Niehaus K, Weisshaar B: Differential regulation of closely related R2R3-MYB transcription factors controls flavonol accumulation in different parts of the Arabidopsis thaliana seedling. Plant Journa/ 2007, 50(4):660-677.

51. Jin H, Cominelli E, Bailey P, Parr A, Mehrtens F, Jones J, Tonelli C, Weisshaar B, Martin C:

Trancriptional repression by AtMYB4 controls production of UV-protecting sunscreens in Arabidopsis. The EMBO Journal 2000, 19(22):6150-6161.

52. He C, Zhang G, Zhang J, Zeng Y, Liu J: Integrated analysis of multiomic data reveals the role of the antioxidant network in the quality of sea buckthorn berry. Faseb Journa/2017, 31(5):1929-1938.

53. Zhou H, Wang KL, Wang F, Espley RV, Ren F, Zhao J, Ogutu C, He H, Jiang Q, Allan AC et al: Activatortype R2R3-MYB genes induce a repressor-type R2R3-MYB gene to balance anthocyanin and proanthocyanidin accumulation. The New phytologist 2019, 221(4):1919-1934.

54. Finn RD, Coggill P, Eberhardt RY, Eddy SR, Mistry J, Mitchell AL, Potter SC, Punta M, Matloob Qureshi1, Sangrador-Vegas A et al: The Pfam protein families database: towards a more sustainable future. Nucleic Acids Research 2015, 44(D1):D279-D285.

55. Crooks GE, Hon G, Chandonia J-M, Brenner SE: WebLogo: a Sequence Logo Generator. Genome Research 2004, 14(6):1188-1190.

56. Bailey TL, Boden M, Buske FA, Frith M, Grant CE, Clementi L, Ren J, Li WW, Noble WS: MEME Suite: tools for motif discovery and searching. Nucleic Acids Research 2009, 37(suppl_2):W202-W208.

57. Guo AY, Zhu QH, Chen X, Luo JC: GSDS: A gene structure display server. Hereditas 2007, 29(8):10231026.

58. Wang Y, Tang H, DeBarry JD, Tan X, Li J, Wang X, Lee T-h, Jin H, Marler B, Guo H et al: MCScanX: a toolkit for detection and evolutionary analysis of gene synteny and collinearity. Nucleic Acids Research 2012, 40(7):e49.

59. Lamesch P, Berardini TZ, Li D, Swarbreck D, Wilks C, Sasidharan R, Muller R, Dreher K, Alexander DL, Garcia-Hernandez $\mathrm{M}$ et al: The Arabidopsis Information Resource (TAIR): improved gene annotation and new tools. Nucleic Acids Research 2011, 40(D1):D1202-D1210.

60. Jaradat MR, Feurtado JA, Huang D, Lu Y, Cutler AJ: Multiple roles of the transcription factor AtMYBR1/AtMYB44 in ABA signaling, stress responses, and leaf senescence. Bmc Plant Biology 2013, 13(1):1-19.

61. Jeong CY, Kim JH, Lee WJ, Jin JY, Kim J, Hong S-W, Lee H: AtMyb56 Regulates Anthocyanin Levels via the Modulation of AtGPT2 Expression in Response to Sucrose in Arabidopsis. Moleculer Cells 2018, 41(4):351-361. 
62. Park J-S, Kim J-B, Cho K-J, Cheon C-I, Sung M-K, Choung M-G, Roh K-H: ArabidopsisR2R3-MYB transcription factor AtMYB60 functions as a transcriptional repressor of anthocyanin biosynthesis in lettuce (Lactuca sativa). Plant Cell Reports 2008, 27(6):985-994.

63. Livak KJ, Schmittgen TD: Analysis of Relative Gene Expression Data Using Real-Time Quantitative PCR and the $2-\Delta \Delta C$ T Method. Methods 2001, 25(4):402-408.

\section{Supplementary Information}

Additional file 1: Table S1. Identification of the 161 R2R3-MYB genes from sea buckthorn in this study. R2R3-MYB transcription factors were named according to the sequence identified from the sea buckthorn genome.

Additional file 2: Table S2. According to the existing references, the number of R2R3-MYB transcription factors in 21 species was calculated and divided into groups.

Additional file 3: Table S3. Amino acid composition of R2 repeat and R3 repeat sequences of R2R3-MYB gene of sea buckthorn.

Additional file 4: Table S4. Conserved motifs identified in R2R3-MYB of sea buckthorn.

Additional file 5: Table S5. The number and order of motifs in the R2R3-MYB genes in our study.

Additional file 6: Table S6. Chromosome location information of the HrMYB transcription factor gene family of sea buckthorn.Table S6 showed the chromosome location of the HrMYB genes, and the distance between the two adjacent genes was calculated according to the starting and ending positions of the gene. The yellow background gene pairs were tandem duplications pairs.

Additional file 7: Table S7. Collinearity relationships of R2R3-MYB genes in sea buckthorn.

Additional file 8: Table S8. The FPKM values of HrMYB genes expression. The 161 HrMYB genes expression value was from root nodule, root, leaf, stem and three stages of fruit.

Additional file 9: Table S9. The quantitative real-time RT-PCR data of 14 HrMYB genes. In 5 different fruit development stages, 14 potential HrMYB expression data related to flavonoid synthesis. Data were normalized to $18 \mathrm{~S}$ rRNA gene.

Additional file 10: Table S10. Sequence information of 14 pairs of HrMYB specific primers for qRT-PCR amplification analysis.

\section{Figures}



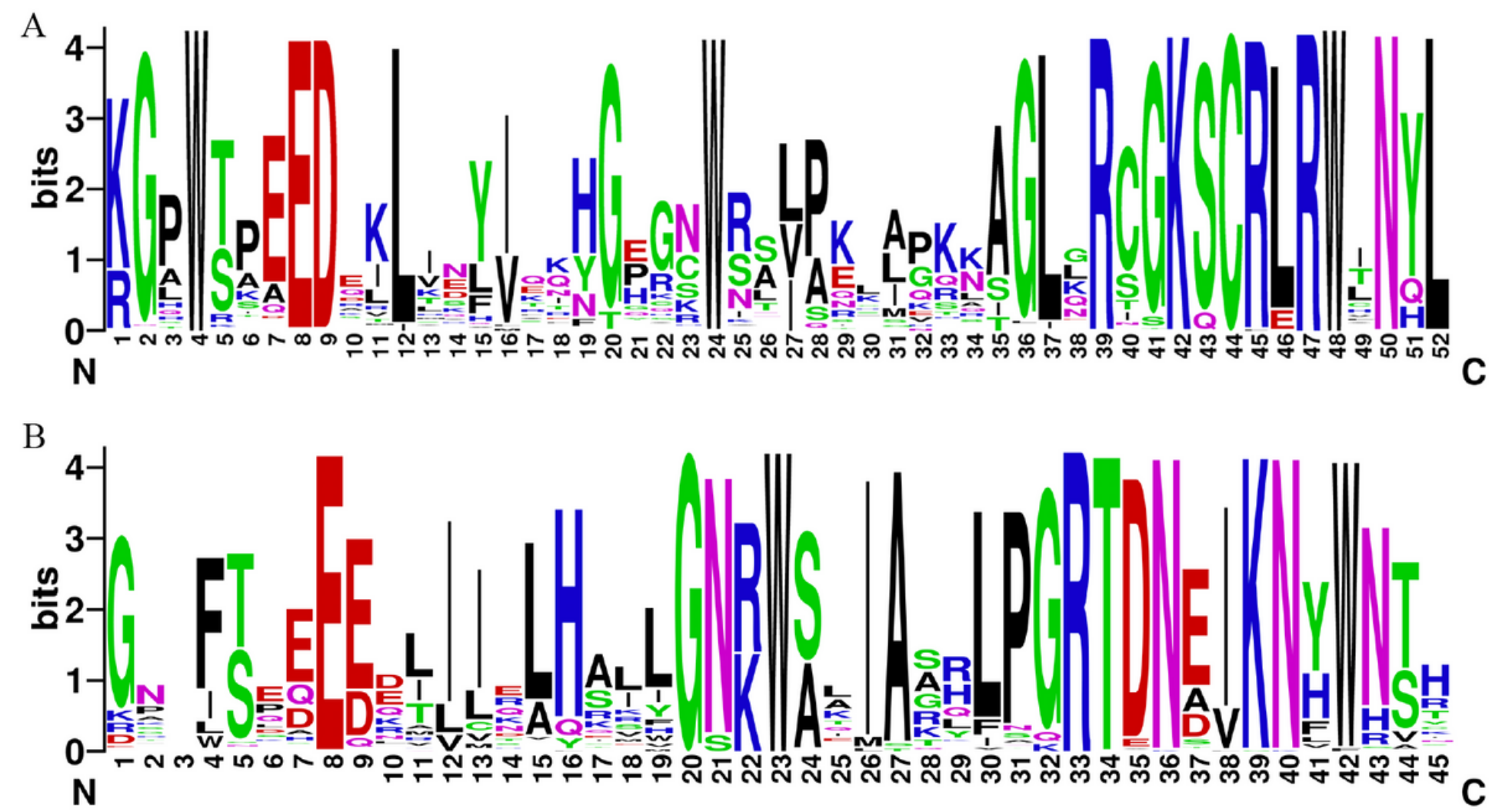

Figure 1

Sequence logos of the R2 (A) and R3 (B) MYB repeats of all sea buckthorn R2R3-MYB domains. The bit score shows the information content for each position in the sequence. Asterisks were used to indicate the highly conserved tryptophan residues (W) in the MYB domain 

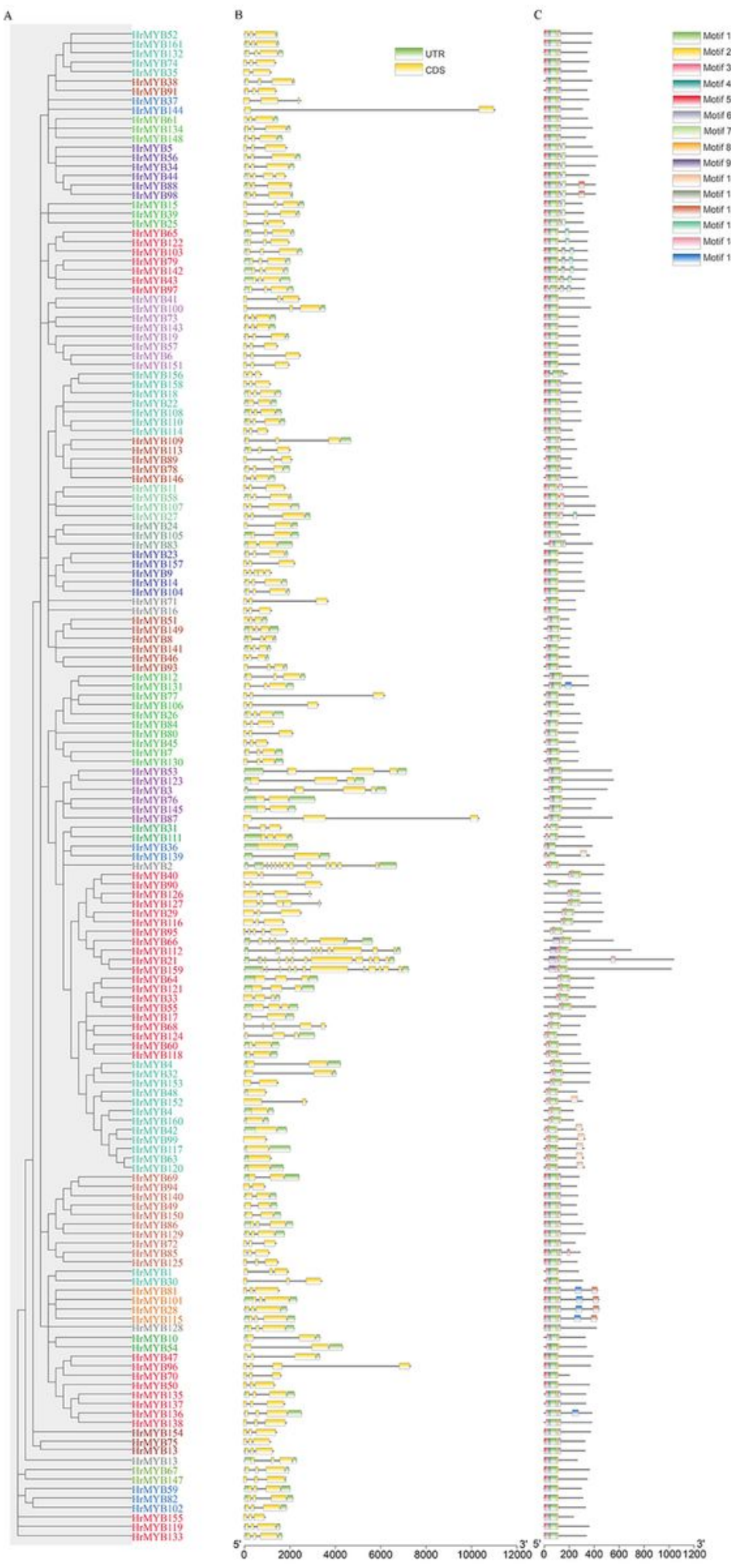

\section{Figure 2}

Phylogenetic relationships, gene structure and architecture of conserved protein motifs in R2R3-MYB genes from sea buckthorn. A The Maximum-Likelihood (ML) tree on the left includes 161 R2R3-MYB proteins from sea buckthorn. The MYB proteins were clustered into 28 subfamilies, sequentially designated as A1 to A28. B Exon/intron structures of R2R3-MYB genes from sea buckthorn. C 
Architecture of conserved protein motifs in 28 subfamilies. Each motif is represented by a number on the colored box.
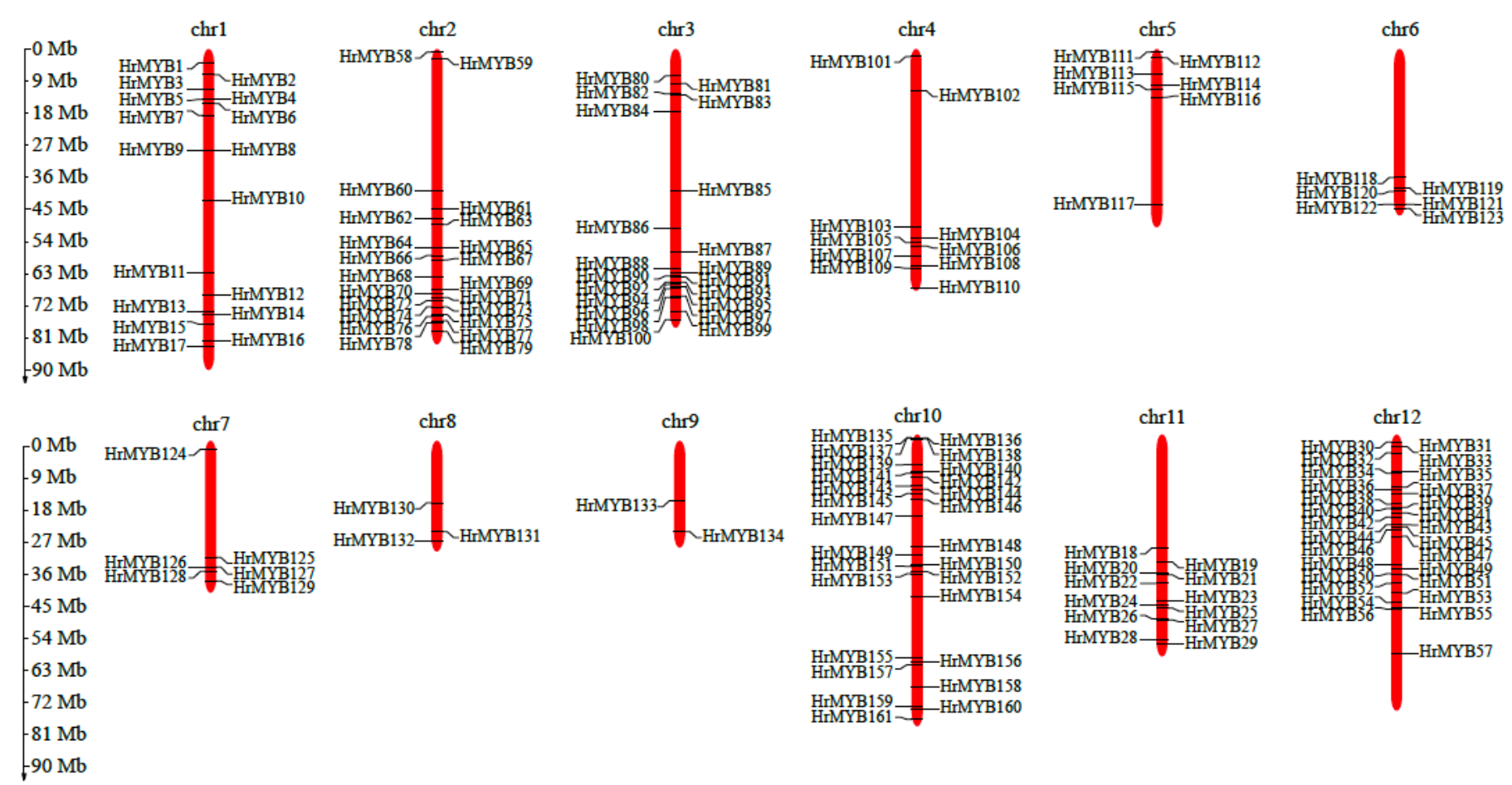

\section{Figure 3}

Chromosomal locations of sea buckthorn R2R3-MYB genes. The scale is in megabases (Mb).

\section{Figure 4}

Schematic representations of interchromosomal relationships of the sea buckthorn R2R3-MYB genes. Gray lines suggest all synteny blocks in the sea buckthorn genome, and the red lines indicate duplicated MYB gene pairs. The chromosome number is indicated at the top of each chromosome 


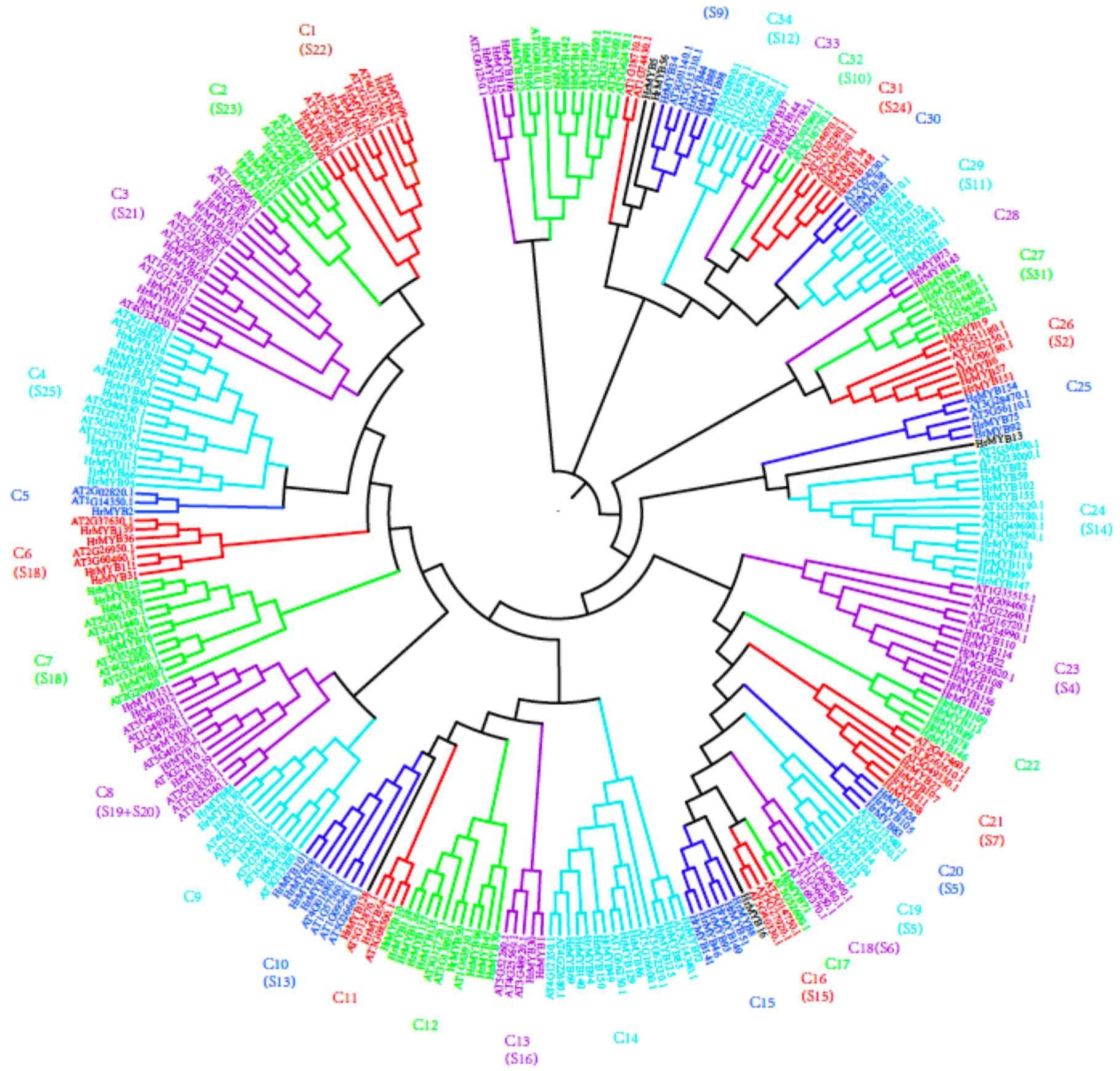

Figure 5

Phylogenetic tree of the sequence of full-length MYB proteins from sea buckthorn and A. thaliana using Maximum-Likelihood method. 


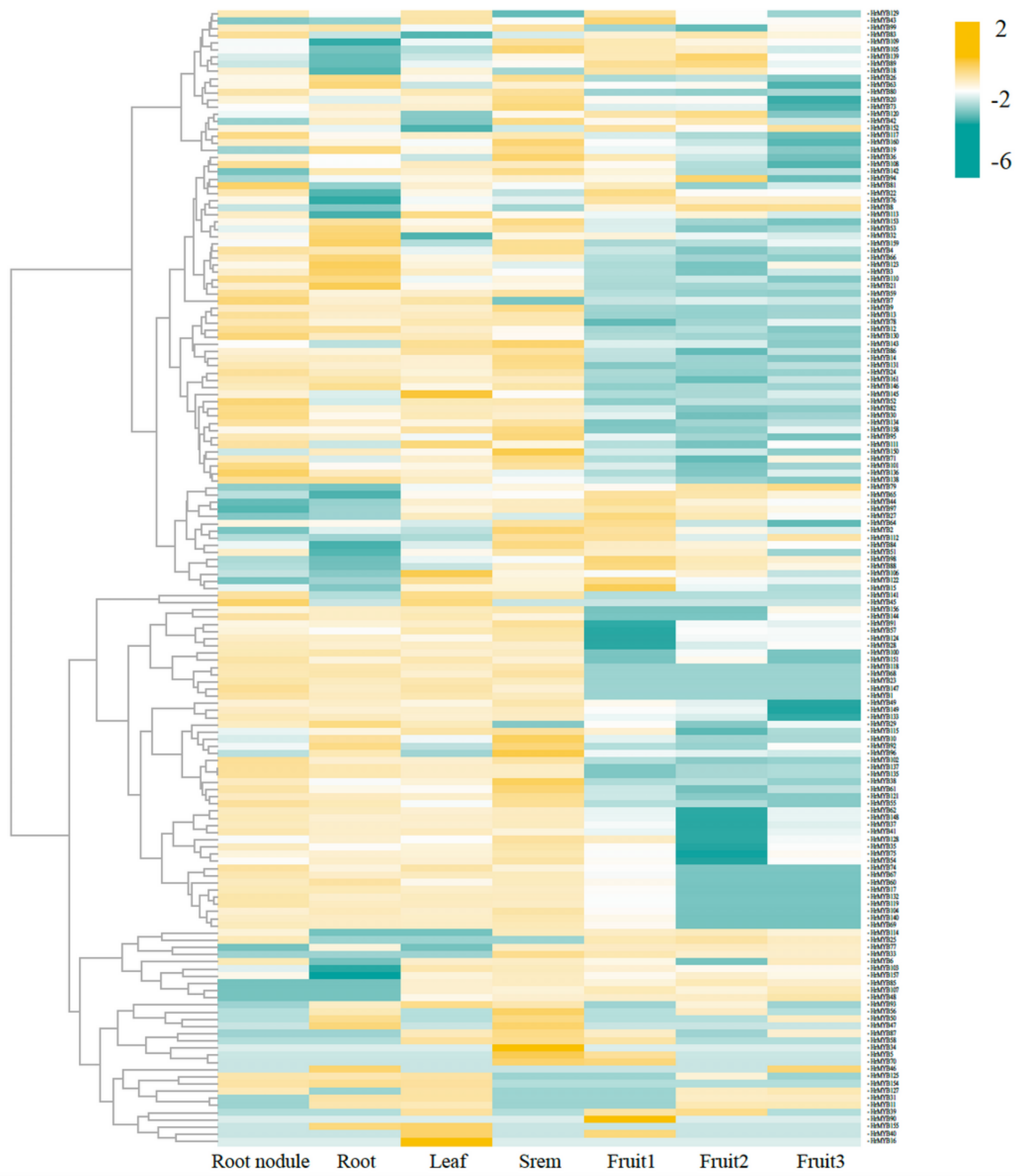

Figure 6

Heat map of HrMYB genes in the root nodule, root, stem, leaf and fruit. Hierarchical clustering results are shown on the left of the heat map and relative expression values with color green (low) to yellow (high) are displayed at the top. 

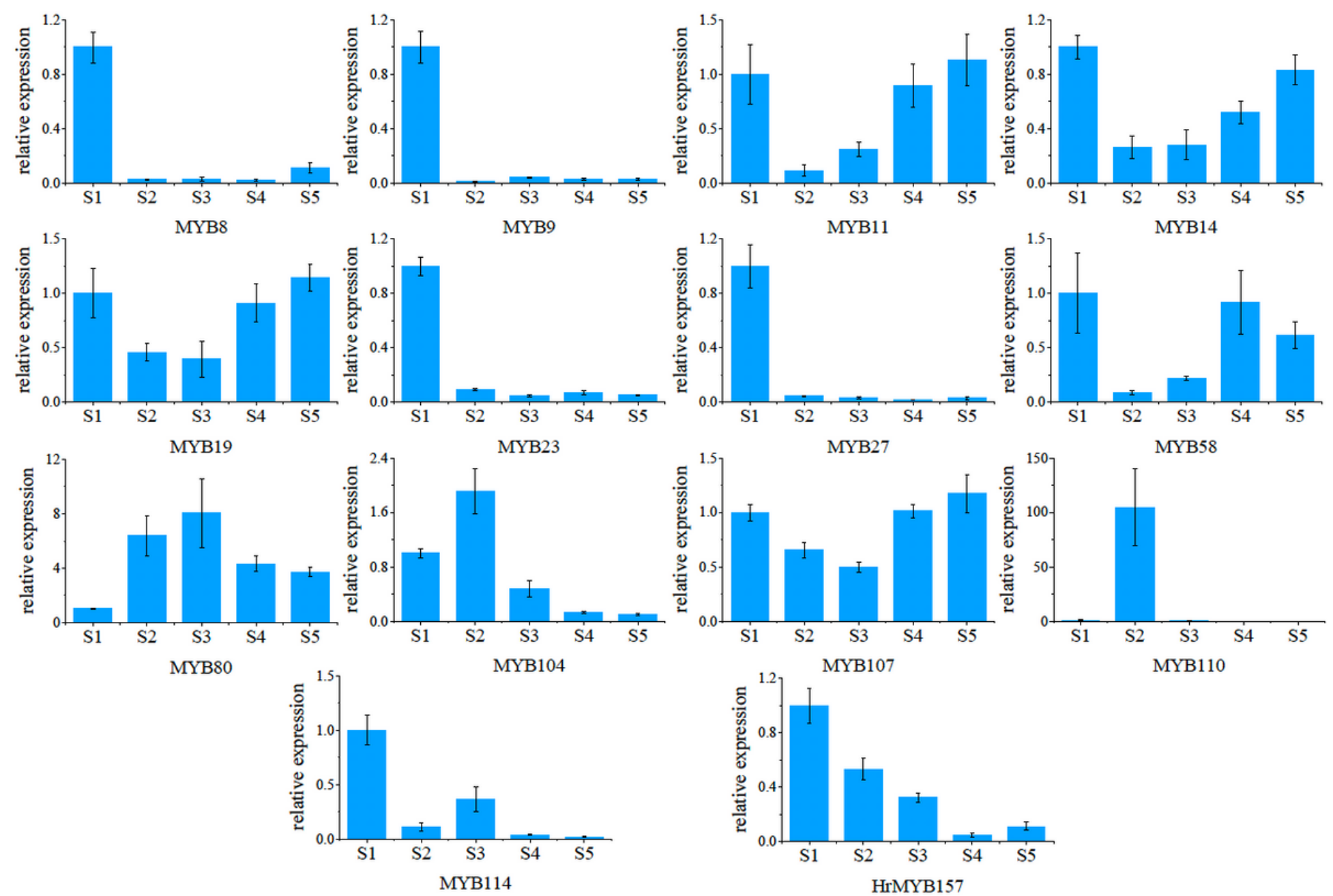

Figure 7

Expression analysis of selected MYB genes using quantitative real-time RT-PCR. Expression patterns of 14 selected HrMYBs involved in flavonoid biosynthesis in different stages of fruit development. Data were normalized to $18 \mathrm{~S}$ rRNA gene and vertical bars indicate standard deviation.

\section{Supplementary Files}

This is a list of supplementary files associated with this preprint. Click to download.

- Additionalfile1TableS1.xlsx

- Additionalfile6TableS6.xIsx

- Additionalfile10TableS10.xlsx

- Additionalfile7TableS7.xlsx

- Additionalfile8TableS8.xIsx

- Additionalfile9TableS9.xlsx

- Additionalfile3TableS3.xIsx 
- Additionalfile4TableS4.xIsx

- Additionalfile5TableS5.xlsx

- Additionalfile2Tables2.xlsx 